\title{
Trends in Mobile Transit Information Utilization: An Exploratory Analysis of Transit App in New York City
}

\author{
Niloofar Ghahramani and Candace Brakewood, Ph.D. \\ The City College of New York
}

\begin{abstract}
Smartphone applications that provide transit information are now very popular. However, there is limited research that examines when and where passengers use mobile transit information. The objective of this research was to perform an exploratory analysis of the use of a smartphone application known as Transit App, which provides real-time transit information and trip planning (schedule) functionality. Backend data from Transit App were examined by time of day and day of week in the New York City metropolitan area. The results show that the pattern of both the trip planning feature and overall realtime information usage follow the typical pattern of transit ridership, which has morning and evening peaks. Additionally, self-reported household locations of Transit App users in the New York City area were compared with household socioeconomic characteristics (specifically, income, ethnicity, and age) from census data using GIS visualizations and the Pearson correlation coefficient, but they do not appear to be correlated. This implies that passengers use Transit App regardless of household income, race, or age trends in their neighborhood. This exploratory study examined a rich new data source-backend data from a transit information smartphone application - that could be used in many future analyses to help transit agencies better understand how transit riders use information and plan their trips.
\end{abstract}

\section{Introduction}

The emergence of smartphones and other mobile-based technologies has revolutionized the way travelers' access both static and real-time transit information. As adoption of these new devices has grown rapidly among transit riders, public transportation agencies have explored the best ways to deliver this information to their passengers. Some transit agencies have developed their own official web or mobile applications, others have released their data openly and encouraged the private sector to develop applications using their data, and some have pursued both strategies. By opening 
their transit data to third parties, agencies have spawned innovative and cost effective applications ("apps") for cities all over the world (Schweiger 2011; Barbeau et al. 2014).

For both transit riders and agencies, easily-accessible real-time information has been one of the main benefits of these applications. When riders know the approximate arrival time of their vehicle, the burden of waiting for transit can be significantly reduced (Watkins et al. 2011; Brakewood et al. 2014; Brakewood, Rojas et al. 2015). For example, bus riders in Seattle, Washington, who used mobile real-time information before arriving at a stop waited approximately two minutes less than other riders; similarly, their perceived wait times were approximately $30 \%$ less than riders who did not use real-time information (Watkins et al. 2011). Additionally, access to real-time information can be influential on an individual's decision to use the transit system. Indeed, prior research has shown that it can increase ridership by approximately $2 \%$ (Tang and Thakuriah 2012; Brakewood, Macfarlane, and Watkins 2015). Considering the constrained budgets of many transit agencies, providing real-time information can be a cost-effective way to increase ridership.

Despite these documented benefits, there has been little prior research examining when and where transit passengers use mobile transit information. Moreover, many of these new information and communication technologies collect detailed data on the backend about when and where this information is being accessed. Therefore, this study aimed to conduct an exploratory analysis of usage of mobile transit information and focuses specifically on Transit App, one of the most popular multi-regional real-time transit information apps. The objective of this research was to analyze trends of Transit App usage by time of day and day of week and to examine the relationship of users' household locations with socioeconomic characteristics in the New York metropolitan area.

\section{Literature Review}

This section provides a brief review of prior research pertaining to real-time transit information. Before the widespread availability of mobile phones, real-time transit information was provided primarily via signage at transit stops or in stations. Many early studies focused on the effects of at-stop signage on transit riders' perceptions and behavior (e.g., Hickman and Wilson 1995; Dziekan and Vermeulen 2006; Dziekan and Kottenhoff 2007; Tang and Thakuriah 2011). More recently, the literature pertains primarily to the passenger and transit agency benefits of providing real-time information via mobile and web-based devices (Zhang et al. 2008; Ferris et al. 2010; Watkins et al. 2011; Tang and Thakuriah 2012; Tang, Ross, and Ha 2012; Carrel et al. 2013; Gooze et al. 2013; Brakewood et al. 2014; Brakewood, Macfarlane, and Watkins 2015). Only two prior references have specifically examined backend data from real-time information transit applications, and these are briefly summarized in the following paragraphs.

The first study using backend data from a real-time transit information smartphone application is an unpublished master's thesis that examined two smartphone applications known as "AnyStop" and "TreKing" (Feakins 2013). The author examined usage patterns in Chicago, Illinois, using a two-week sample from December 2010 from 
AnyStop and a three -month sample from 2011 from TreKing. By counting the number of sessions recorded in the AnyStop backend database by time of day, the author concluded that the pattern of using AnyStop followed the classic pattern of transit ridership: on weekdays, there are two significant peaks in the morning and evening and one smaller peak around noon, and on weekends, there are multiple peaks distributed relatively equally throughout the day. The author also compared the utilization of both apps to route-level Chicago Transit Authority ridership data and found correlation between the usage of these transit apps and ridership levels (Feakins 2013).

The second and more recent study used backend data from a real-time information transit application called Transit App, which is also the focus of this paper (Davidson 2016). Davidson (2016) examined similarities and differences of origin-destination patterns between two datasets. One dataset was the trip planning feature of Transit App, which provides a-to-b directions based on transit schedules, and the other was the most recent (2010/2011) Regional Household Travel Survey conducted by the New York Metropolitan Transportation Council. The trip planning feature of Transit App provides origins and destinations of the trips that app users are interested in making, whereas the Regional Household Travel Survey data provides the stated origins and destinations of actual trips. The two datasets were compared at the community board level, and the results suggest that they have very similar origin-destination patterns (Davidson 2016).

This brief literature review demonstrates that there has been limited research using the backend data from transit information apps. The most relevant prior study looked at the same data source and location as this study (Transit App backend data in New York City), but it considered only the trip planning feature of the app, which provides scheduled transit information. The analysis in Davidson's study consists of a small subset of the data; real-time information and temporal differences were not studied. The other relevant prior study used Chicago data from 2010 and 2011; however, adoption of smartphones has rapidly grown since then, which could lead to different trends. Moreover, there has been little analysis of who is actually using these apps. Therefore, this study sought to fill these gaps in the literature by examining a recent real-time information dataset and assessing socioeconomic trends associated with usage.

\section{Objective}

The objective of this study was to perform an exploratory analysis of Transit App usage among transit riders in the New York metropolitan region. The specific topics addressed in this study fall into two categories: general Transit App utilization patterns and usage among different socioeconomic groups. The first section explores overall Transit App usage by time of day and day of week and analyzes whether individual users can be categorized by their daily frequency of app usage. The second part examines whether Transit App may be used more frequently in areas with residents of certain socioeconomic characteristics. 


\section{Dataset}

This section provides a brief description of the data used in this analysis. First, background information is provided about Transit App, and next, the geographic area of analysis is defined. Last, a detailed description of the data files used in the analysis is provided.

\section{Overview of Transit App}

Transit App is a company based in Montreal, Canada, that has developed a freelyavailable smartphone application providing urban transportation information. In 2012, the company released the first version of its application for iPhone. In the initial version, the app provided transit schedule information for Montreal, Toronto, and Quebec City. Since then, Transit App launched an Android version of its application and has expanded to more than 100 cities in 9 countries, including widespread coverage in the United States. Transit App also has added many features, including real-time transit information, trip planning using schedule information, service alerts, and multimodal support (including bike sharing, car sharing, and Uber). The most heavily-used features of the app are those providing real-time transit information, and Transit App uses realtime information to display transit vehicle departure times when the transit agency makes it available; otherwise, it displays schedule information (Transit App 2015). Additionally, users can store their favorite locations in the app, such as home or work, to facilitate quickly finding information that they commonly use. The app includes a combination of map and list view interfaces. Figure 1 shows the Transit App Android interface displaying real-time transit information for nearby routes (left), trip planning (center), and a stored home location (right), respectively.

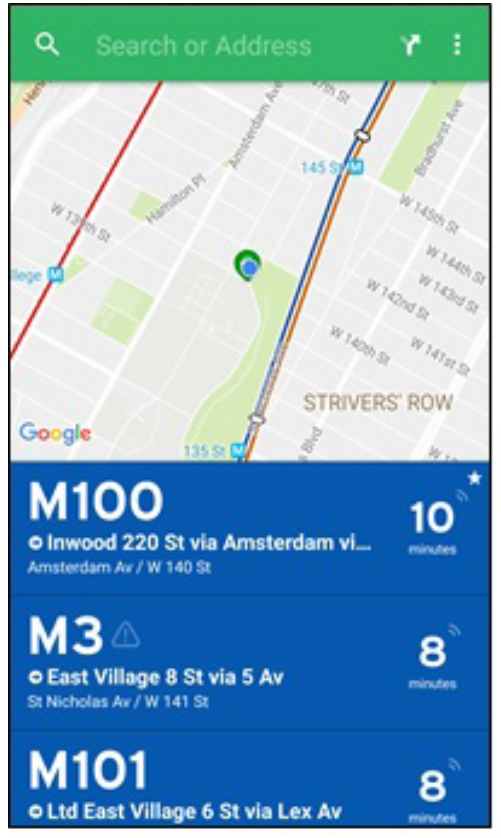

a) Nearby routes

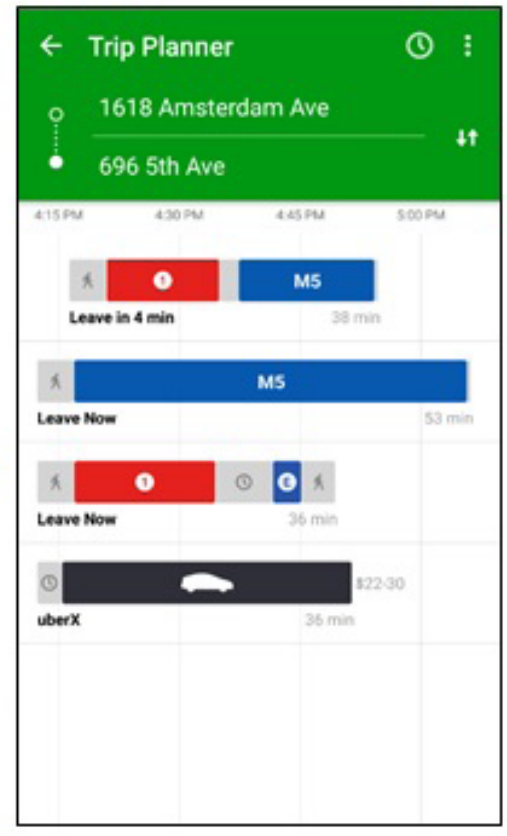

b) Trip planning

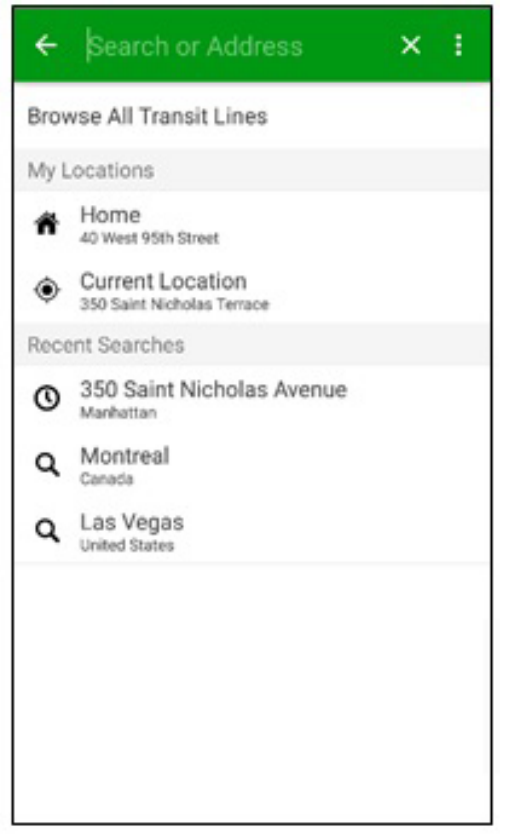

c) a stored home location

FIGURE 1. Transit App screenshots 


\section{Area of Analysis}

The New York metropolitan area was selected as the geographic area for this analysis because it has the highest concentration of transit trips in the United States (McKenzie and Rapino 2011) and also has the highest Transit App usage in the United States. Because smartphones are mobile devices and some people used the app in regions other than New York, a small percentage of Transit App sessions in this dataset took place in other regions. Usage outside the New York region was not considered in this analysis and was removed from the dataset before conducting the following analysis. After removing the records from outside the New York region, these datafiles, which are discussed in the following section, were imported into ArcGIS 10-2.

\section{Data Files and Description}

The dataset for this study was obtained directly from Transit App and contains data for any user that opened Transit App at least once in October 2014 in the New York City region. The dataset includes the user location (latitude/longitude), which is recorded whenever the application is opened. For privacy purposes, all geographic coordinates contained in these files were offset by Transit App developers by a random number up to 300 meters per position. By anonymizing the data, Transit App ensured that none of the data used in this analysis contain personally identifiable information. Also, locations mentioned herein refer to the anonymized version of the data point (e.g., a reference to "home locations" refers to the anonymized home locations).

The raw dataset contained multiple files in a Comma Separated Values (CSV) format, which were as follows:

- Locations file: Every time users open Transit App, regardless of what feature they are using, their location is sent to the Transit App server based on the coordinates from the location services in their smartphone. Date, time, accuracy of their location, and speed (e.g., if they are in a vehicle) are recorded in what is referred to as the "locations" file. Also, a unique session ID is created each time a user opens the app. For October 2014, a total of 10,875,013 records were sent to the Transit App server for the New York metropolitan area. This file was imported into GIS based on the users' start coordinates (i.e., where they were when opening Transit App).

- Trips file: This file contained information about usage of the trip planning feature in Transit App and is referred to as the "trips" file. Specifically, it included start and end coordinates (latitude/longitude), date, and timestamps of all the trip planning requests. It should be noted that this is a subset of the locations file because anytime a user opens the app, including using the trip planning feature, his/her coordinates are stored in the locations file. For October 2014, the trips file had a total of 399,831 records for the New York metropolitan area. This file was imported into GIS based on the users' start coordinates (i.e., where they were when opening Transit App). 
- Placemarks file: This file included coordinates of home and work locations that users have stored in Transit App and is referred to as the "placemarks" file. This represents data from an optional function in Transit App where users can store places that they often go (e.g., home or work) to easily access relevant transit information for that specific location. This file contains the coordinates of users' home or work locations, of which there were a total of 11,782 in New York metropolitan area. Of these, only the home locations were imported into GIS based on the user's defined coordinate.

\section{Analysis of General Utilization Trends}

For this analysis, nine counties in New York and nine counties in New Jersey were included in the area of analysis; counties farther than Somerset and Morris counties in New Jersey had few Transit App users and were excluded. North of New York City, Westchester and Rockland counties were selected as the New York boundaries since there were very few Transit App users in counties farther north and in counties located in Connecticut. From the east, Suffolk County was set as the boundary.

For this analysis of general trends, Transit App usage was first examined by time of day and then by day of week. Next, the frequency of usage in a day by individual app users was examined. For each of these analyses, the overall usage of Transit App (which is predominantly checking real-time arrival information) and the utilization of only the trip-planning feature (for a-to-b directions) were considered separately

\section{Time of Day Analysis}

First, the data were categorized by date and hour. Using the open source statistical program RStudio (RStudio Team 2015), the number of unique session IDs were counted in each hour for the two datasets (all records from the locations file and the trip planning feature only); the results are shown in Figure 2. It should be noted that the scales on the $y$-axis of Figure 2 are different for the graph displaying all usage from the locations file and the trip planning feature because the number of unique sessions is much larger for all usage. 

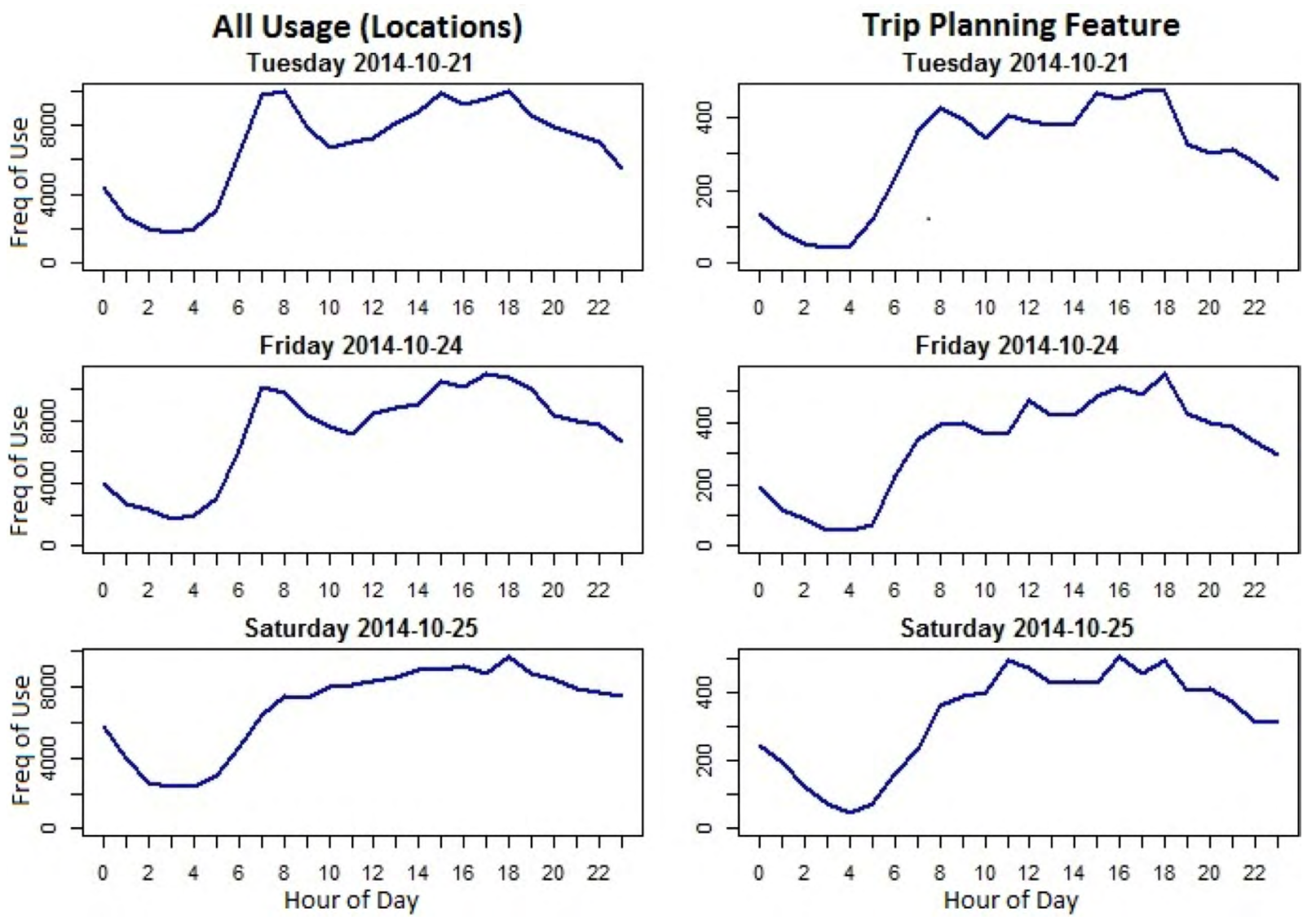

FIGURE 2. Frequency of using Transit App by time of day

On Tuesday, October 21, 2014, there were approximately 178,000 unique sessions in the locations file and only approximately 7,000 unique session IDs in the trips file. Although Tuesday was selected for presentation, this pattern was typical of Mondays, Tuesdays, Wednesdays, and Thursdays. Both October 21 graphs have two significant peaks: one in the morning from 6:00-8:00 $A M$ and another in the evening from 3:00-6:00 PM, which likely represent commuting trips. There is also a smaller peak around 12:00 noon, which may represent trips for lunch or personal business. Another interesting finding is the slightly higher and wider range of Transit App usage in the evening peak period compared to the morning peak. This disparity may be because users make more recreational trips after work or chain errand/shopping trips in the evening. It also may imply that users do not use the app as frequently in the morning, perhaps because they are more familiar with their morning commutes.

For Friday, October 24, 2014, the pattern of Transit App usage shows overall usage of approximately 179,000 unique sessions and use of the trip planning feature of approximately 7,800 unique sessions. Both Friday graphs reveal morning and evening peaks similar to the Tuesday image. However, the evening peak on Fridays is slightly higher and wider (from 3:00-7:00 PM) than the evening peak on Tuesdays. This larger peak may represent additional leisure trips or people leaving work earlier on Friday afternoons. 
For Saturday, October 25, 2014, the pattern of Transit App usage for all features includes approximately 164,000 unique sessions and the trip planning feature includes approximately 7,700 unique sessions. The Saturday figures do not have large peaks; instead, multiple small peaks are distributed nearly equally throughout the day, possibly for recreational and leisure trips. Additionally, usage on Saturday was lower than on weekdays, which is in line with lower levels of transit ridership generally seen on weekends.

\section{Day of the Week Analysis}

To compare usage frequency of Transit App on different days of the week, the total number of unique sessions for each day were counted for overall usage of Transit App as well as for the trip planning feature. Figure 3 shows total usage and trip planning utilization for Sunday, October 19, 2014, to Saturday, October 25, 2014. Total usage ranged from 165,000 to 185,000 unique sessions per day for the New York City region, whereas there were only 6,600 to 7,800 unique trip planning sessions per day. The peak for all usage occurred on Monday, whereas trip planning utilization was highest on Friday; the high value on Friday may be because of additional non-commute trips (e.g., recreational trips) for which travelers seek trip planning information. On Sunday, usage (both overall and trip planning specifically) was slightly lower, which likely reflects dayof-the-week trends in transit ridership.

FIGURE 3.

Use of Transit App by day of week, October 19-25, 2014
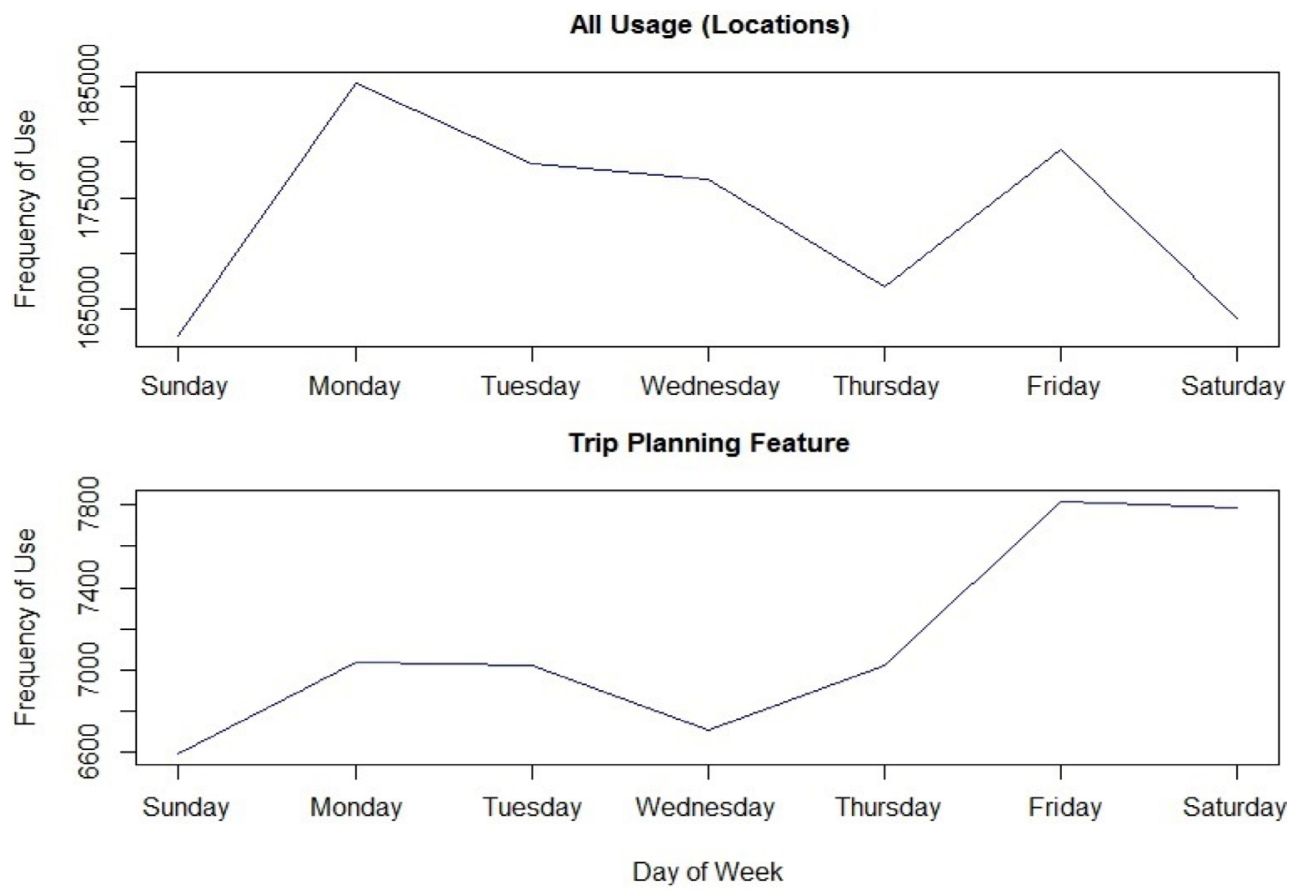

Individuals' Frequency-of-Use Analysis

This analysis examined how often individual Transit App users typically check the app in a single day. The number of sessions associated with each user defined by their unique device ID was counted, and the results are shown for a single day (Tuesday, October 21) 
in Figure 4. Results for all usage from the locations file indicate approximately 22,000 users, and trip planning usage had approximately 3,600 users. As can be seen in Figure 4 , frequency of usage from the locations file shows that almost $80 \%$ of users checked the Transit App two times or more in one day. This may imply that many Transit App users check the app for their commute to and from work and may be accustomed to checking the app for most, if not all, of their daily trips.

FIGURE 4.

Frequency of Transit App usage by individuals on Tuesday, October 21, 2014

\section{All Usage (Locations)}

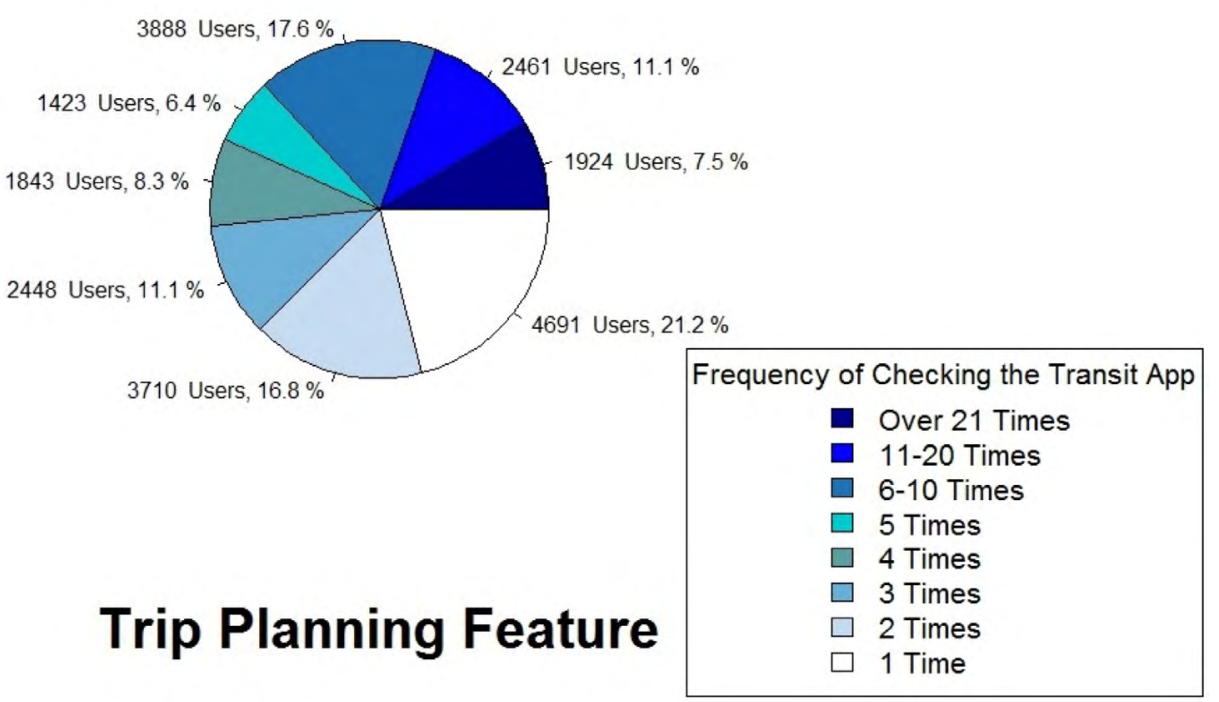

The percentage of users who checked Transit App exactly once per day is almost three times higher for the trip planning feature (59.3\%) than for the overall utilization (21.1\%). Infrequent users of the trip planning feature may represent either infrequent users of the transit system, travelers making irregular trips, or those who use the app for only one way of a commute trip.

Notably, there was a small number of heavy users (i.e., more than 21 times in a day), which may have been the result of experiencing delays on the transit system. These sessions most likely represent "simulated" sessions, meaning that the user moved the GPS point on the map interface of the app to a location other than where they actually were to search for transit information there. Alternatively, these heavy users may not 
have exited the app, so it was running in the background on their phones and sending signals to the server. Further investigation of these heavy users is recommended for future research.

This analysis was repeated for other days in October 2014, which showed similar patterns.

\section{Census Analysis}

The next set of analyses assessed whether Transit App is used more frequently in areas with residents of certain socioeconomic characteristics (specifically, income, race, and age). For the analysis in this part, nine counties in New Jersey and five counties in New York (specifically, the five boroughs of New York City) were considered. The geographic unit chosen for this analysis was the census tract, and tract geometry was obtained from the US Census Bureau website (United States Census Bureau 2015). Socioeconomic information was obtained from the 2010 census through the American Fact Finder website (United States Census Bureau 2015). Because census data are based on home locations, the self-reported home locations of Transit App users were chosen to represent app utilization for this analysis. The home locations of Transit App users were imported into GIS and joined to census tracts based on their coordinates. The number of home locations was counted for each tract and compared to the population density in each tract from the census data. The number of home locations for each census tract was then compared with mean income, dominant race, and dominant age from the census data. The results are discussed in the following sections.

\section{Transit App Users' Home Locations and Population Density}

Transit App users' self-reported home locations and population density in census tracts across the five boroughs of New York and areas of New Jersey are shown in Figure 5. Transit App users' home locations appear to be distributed relatively equally among the Bronx, Manhattan, and Staten Island. In Manhattan, home locations have a slightly higher frequency on the west side of Manhattan. Also, the census tract including Central Park has the highest observed value of Transit App users' home locations (29 in total); this is likely due to the shift applied to the latitude and longitude by Transit App developers to respect user privacy since there is very high residential density on either side of Central Park. Staten Island has a relatively low number of Transit App users' home locations (fewer than 5 homes in most census tracts). Many of the census tracts in Queens with higher frequency of Transit App home locations are located near subway lines. In Brooklyn, the number of home locations in each census tract is higher in the areas closer to Manhattan and Queens (mainly 6-20 home locations per tract) and lower in the southern parts of Brooklyn (fewer than 5 home locations in each census tract). In New Jersey, Transit App users' home locations are mostly concentrated on the eastern part of the state close to Manhattan; many of the census tracts with higher numbers of Transit App users' homes are located near the Port Authority Trans-Hudson (PATH) lines and NJ TRANSIT's light rail lines. 


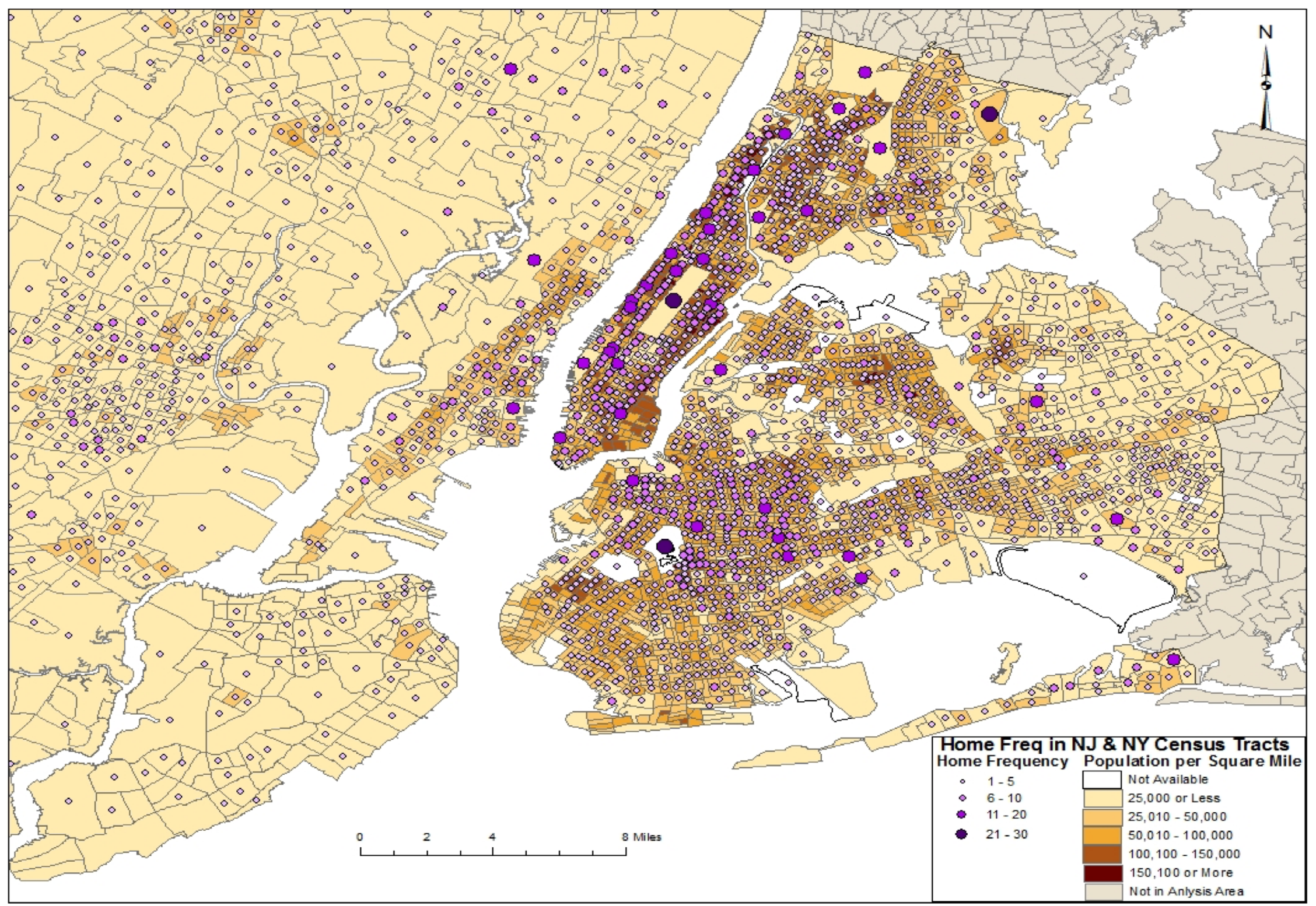

FIGURE 5. Population density and Transit App users' home locations in New York and New Jersey census tracts 
Figure 5 also shows that population per square mile is relatively low (less than 25,000 people per square mile) and relatively homogeneous throughout the census tracts in New Jersey and Staten Island. Population density is higher and more diverse across census tracts in Brooklyn, Queens, and the Bronx. In these three boroughs, population density generally ranges from 25,000 to 100,000 people/square mile. The highest population density is observed in Manhattan's census tracts, which generally fall in the range of 50,000 to 150,000 people/square mile. Distribution of Transit App users' home locations and population density also shows that in the census tracts with higher population density, the number of Transit App users' home locations is also higher, which is an expected observation.

\section{Income Visualization}

Figure 6 shows Transit App users' home locations in relation to mean annual household income per census tract in the five boroughs of New York and areas of New Jersey. As can be seen in this figure, higher income levels are observed primarily in Manhattan (except the northern part). In the other boroughs, mean household income is lower in the majority of census tracts. Many census tracts have a mean annual household in the range of $\$ 40,000$ to $\$ 80,000$ in Brooklyn, the Bronx, and Queens. In Manhattan, the Bronx, and Staten Island, Transit App users' home locations are distributed relatively equally. In New Jersey, Queens, and Brooklyn, frequency of home locations is slightly higher in the areas with higher accessibility to other boroughs or higher concentrations of rail service. Therefore, based on graphical observations of mean household income, Transit App usage does not seem to be associated with household income levels in the New York City area, since Transit App users live in both high- and low-income areas.

\section{Race Visualization}

Figure 7 shows dominant race from the 2010 census data overlaid with the Transit App users' home locations. In Manhattan and Staten Island, the dominant race in most of the census tracts is white, except in the north of Manhattan. In these two boroughs, Transit App users' home locations are distributed relatively equally among the census tracts. In the Bronx, most census tracts have a dominant race of Hispanic/Latino or Black/African American. In Brooklyn, many census tracts in the northern part of the borough have a dominant race of Black/African American, and many census tracts in the south part have the dominant race of white. In Queens, the dominant race in the southern census tracts is Black/African American; in the other parts of Queens, most census tracts have a dominant race of white. The majority of census tracts in New Jersey have the dominant race of white except those close to Manhattan. In terms of Transit App users' home locations, there appears to be little, if any, relationship between dominant race and Transit App users' home locations in the New York City region based on this visualization. 


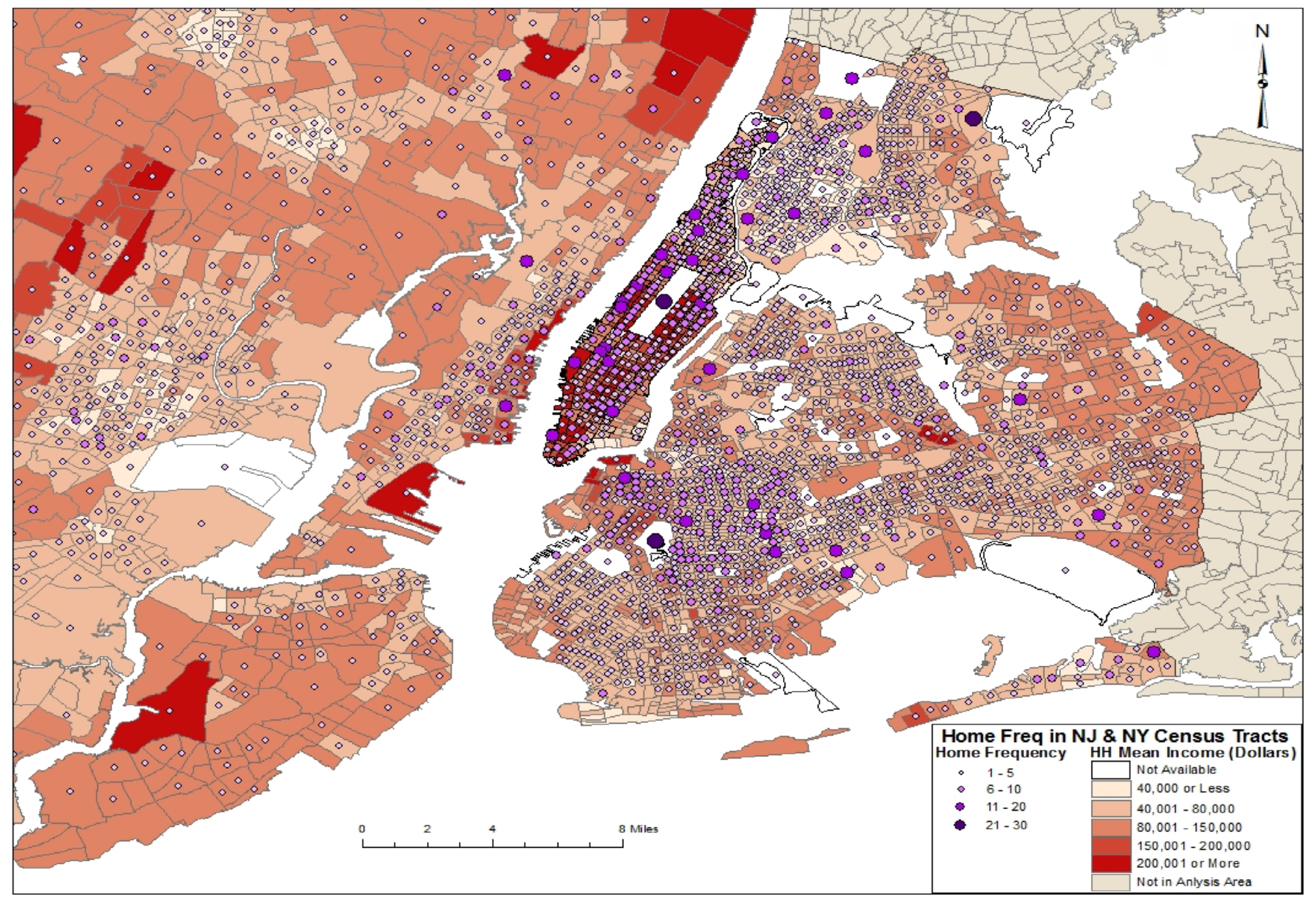

FIGURE 6. Mean annual household income and Transit App users' home locations in New York and New Jersey census tracts 


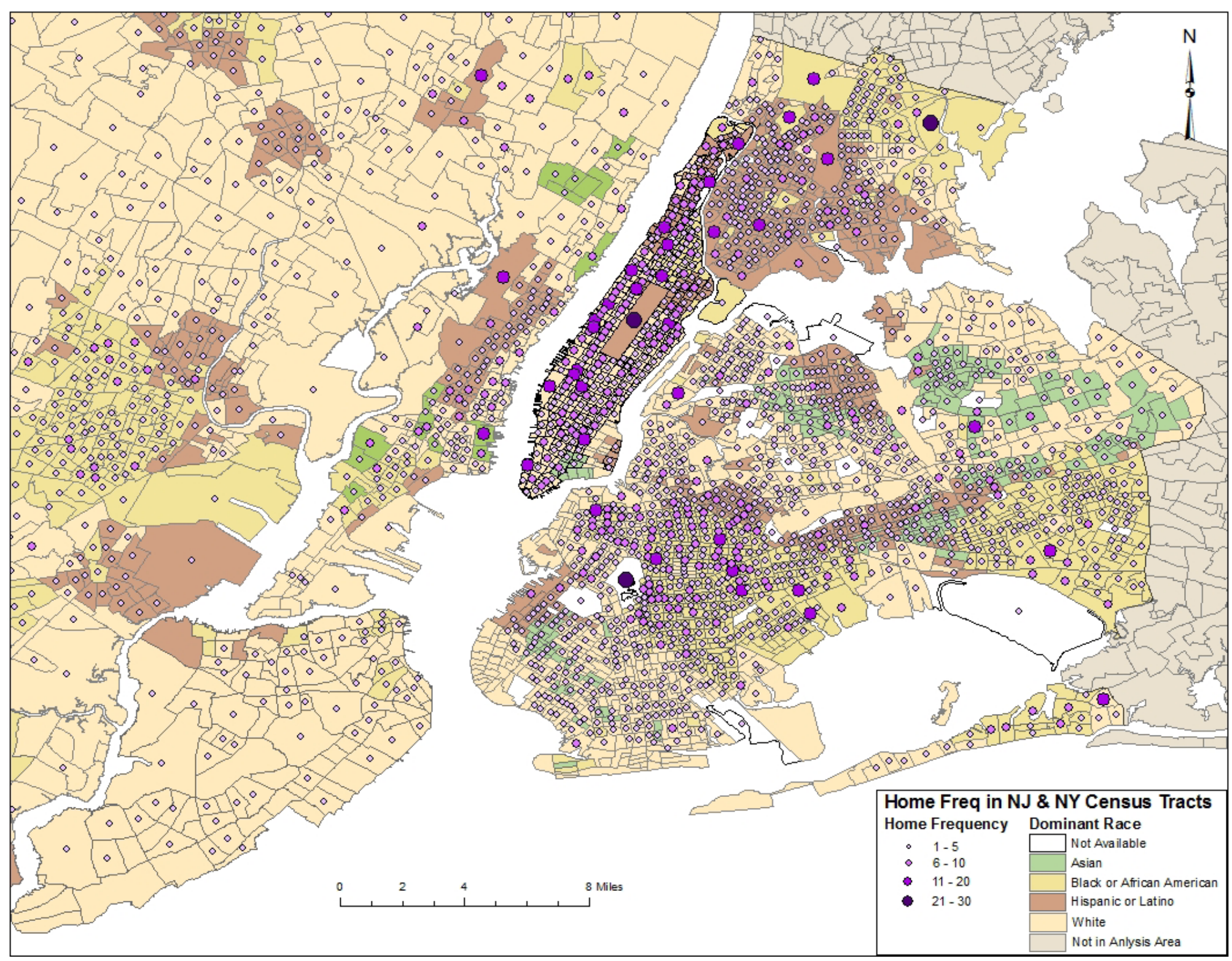

FIGURE 7. Dominant race and Transit App users' home locations in New York and New Jersey census tracts 


\section{Age Visualization}

Figure 8 shows dominant age from the 2010 census data and the number of Transit App users' home locations per census tract. In Manhattan and Staten Island, the majority of the census tracts have relatively homogeneous dominant age group patterns; in Manhattan, the dominant age typically falls in the range of 25 to 34, and in Staten Island, it falls in the range of 45 to 54. Transit App users' home locations are distributed relatively equally in these two boroughs. Therefore, it is unclear if there is a relationship between age and frequency of using Transit App in these two boroughs. Distribution of dominant age is more diverse across the Bronx, Queens, and New Jersey census tracts. In these areas, the visualization does not show much, if any, relationship between age and Transit App users' home locations. However, in Brooklyn, many of the census tracts with more than six Transit App users' home locations fall in the dominant age range of 25 to 34 , so age may play a role in influencing Transit App utilization in this borough.

\section{Statistical Analysis}

In addition to the visualizations presented in the previous section, a statistical analysis also was conducted to understand the relationship between household level socioeconomic characteristics from the census data and Transit App users' home locations. This analysis was performed at the census tract level in nine counties of New Jersey and five counties of New York. Table 1 shows the total number of census tracts, total number of the Transit App users' home locations, and the number of census tracts with at least one Transit App user's home location in each county considered for this analysis. 


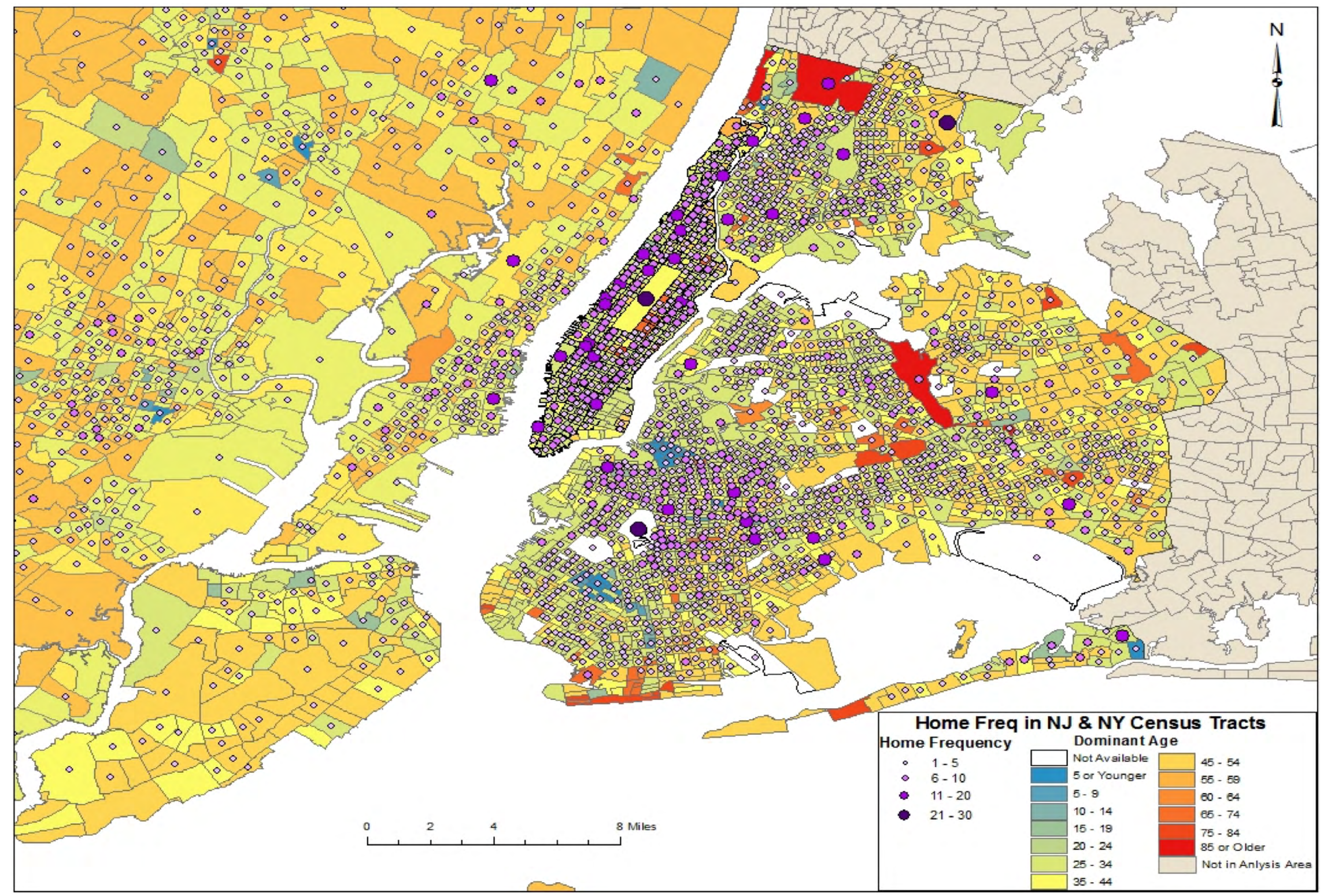

FIGURE 8. Dominant age and Transit App users' home locations in New York and New Jersey census tracts 
TABLE 1.

Number of Census Tracts and Transit App Users' Home Locations

\begin{tabular}{|l|c|c|c|}
\hline Geographic Area & $\begin{array}{c}\text { Total Number of } \\
\text { Transit App Users' } \\
\text { Home Locations }\end{array}$ & $\begin{array}{c}\text { Number of Census Tracts } \\
\text { with at Least One Transit } \\
\text { App Users' Home Location }\end{array}$ & $\begin{array}{c}\text { Total Number } \\
\text { of Census } \\
\text { Tracts }\end{array}$ \\
\hline Manhattan & 1,258 & 253 & 288 \\
\hline Brooklyn & 2,014 & 592 & 761 \\
\hline Bronx & 906 & 280 & 339 \\
\hline Queens & 1,462 & 542 & 669 \\
\hline Staten Island & 126 & 65 & 110 \\
\hline New Jersey (9 counties) & 1,675 & 684 & 1,250 \\
\hline
\end{tabular}

To understand the relationship between household level socioeconomic characteristics obtained from census data and the number of Transit App users' home locations in each census tract, the Pearson correlation coefficient was calculated for each borough in New York City and for northern New Jersey. The Pearson correlation coefficient is a mathematical indicator of the relationship between two variables, and it ranges from - 1 to +1 , with -1 indicating total negative correlation, zero indicating no correlation, and +1 indicating total positive correlation. The Pearson correlation coefficient was calculated between the ratio of Transit App users' home locations to total population and mean household income, percentage of different age groups or percentage of different races in each census tract using RStudio.

The results of Pearson correlation analysis are shown in Table 2. Mean annual household income is negatively correlated with the number of Transit App users' home locations divided by the total population in three of the areas (Manhattan, the Bronx, and New Jersey). The correlation value is relatively small ( $r \sim-0.2$ in New Jersey; $r \sim-0.01$ in Manhattan and the Bronx), implying that Transit App usage and mean household income are weakly dependent and that those in areas with higher income levels may use the Transit App less. In Staten Island, the observed correlation was positive ( $r$ 0.5), which may be because of the low and equally-distributed Transit App usage across Staten Island's census tracts. There are fewer than five Transit App users' home locations in most of Staten Island's census tracts. The Pearson correlation coefficient showed little, if any, relationship for Brooklyn and Queens $(r<0.01)$.

Among different age groups, in Manhattan, Brooklyn, and New Jersey, the percentage of the population in the age range of 20 to 34 had slightly positive correlation with the number of Transit App users' home locations divided by the total population. Similarly, in the Bronx, Brooklyn, Queens, and Staten Island, the percentage of the population in the age range of 35 to 54 had slightly positive correlation with the number of Transit App users' home locations divided by total population. Many of the lowest correlation values were observed in the age groups of 55 to 64 and 65 or older. This suggests that areas with higher percentages of older residents may be less likely to use Transit App.

In the terms of ethnicity, correlation values between different race groups and the number of Transit App users' home locations divided by the total population were very small (close to zero) across all boroughs. The only exceptions were in New Jersey, where the percentage of Transit App users' home locations has a positive correlation with percentage of Black/African American population ( $r \sim 0.358)$, and in Staten Island, 
where percentage of Transit App users' home locations has a positive correlation with the percentage of Hispanic/Latino population $(r \sim 0.334)$. Altogether, correlation values suggest that there is limited correlation between ethnicity and use of the Transit App as represented by user's home locations.

TABLE 2. Pearson Correlation Coefficient Results*

\begin{tabular}{|l|c|c|c|c|c|c|}
\cline { 2 - 6 } \multicolumn{1}{c|}{} & $\begin{array}{c}\text { Manhattan } \\
\text { Census Tracts }\end{array}$ & $\begin{array}{c}\text { Bronx } \\
\text { Census Tracts }\end{array}$ & $\begin{array}{c}\text { Brooklyn } \\
\text { Census Tracts }\end{array}$ & $\begin{array}{c}\text { Queens } \\
\text { Census Tracts }\end{array}$ & $\begin{array}{c}\text { Staten Island } \\
\text { Census Tracts }\end{array}$ & $\begin{array}{c}\text { New Jersey } \\
\text { Census Tracts }\end{array}$ \\
\hline Mean Household Income & -0.011 & -0.017 & 0.005 & 0.009 & 0.497 & -0.215 \\
\hline Percent Under Age 5 & -0.098 & -0.199 & -0.092 & -0.024 & -0.208 & 0.196 \\
\hline Percent Ages 5-14 & -0.232 & -0.251 & -0.092 & -0.199 & 0.535 & -0.142 \\
\hline Percent Ages 15-19 & -0.033 & -0.170 & -0.020 & -0.150 & -0.221 & -0.060 \\
\hline Percent Ages 20-34 & 0.010 & -0.309 & 0.242 & -0.223 & -0.017 & 0.361 \\
\hline Percent Ages 35-54 & -0.346 & 0.278 & 0.013 & 0.194 & 0.298 & -0.107 \\
\hline Percent Ages 55-64 & 0.150 & -0.200 & -0.140 & 0.349 & -0.286 & -0.218 \\
\hline Percent Ages 65 and Over & 0.338 & 0.384 & -0.106 & -0.009 & -0.218 & -0.212 \\
\hline Percent of White Population & 0.093 & -0.087 & -0.142 & 0.062 & 0.041 & -0.418 \\
\hline Percent of Asian Population & 0.017 & -0.053 & -0.133 & -0.071 & -0.032 & 0.030 \\
\hline $\begin{array}{l}\text { Percent of Hispanic/Latino } \\
\text { Population }\end{array}$ & -0.124 & -0.097 & 0.018 & -0.074 & 0.334 & 0.145 \\
\hline $\begin{array}{l}\text { Percent of Black/African } \\
\text { American Population }\end{array}$ & -0.075 & 0.151 & 0.167 & 0.022 & -0.018 & 0.358 \\
\hline
\end{tabular}

*Pearson correlation coefficient results for socioeconomic characteristics and number of Transit App users' home locations divided by total population

\section{Conclusions and Future Research}

Transit App is one of the most popular mobile applications in North America that provides real-time transit information and a-to-b directions based on transit schedules. This study used backend data from Transit App to examine when and where passengers use this information in the New York City metropolitan area in a two part analysis, and the key findings are summarized as follows.

General trends of Transit App usage were first assessed by time of day and day of week. The time of day analysis for overall utilization and the trip planning feature showed that there were two significant peaks-one in the morning and the second in the eveningfor Monday through Thursday, which likely represents the typical pattern of transit ridership. On Fridays, the evening peak was slightly higher and wider than the evening peak on Mondays through Thursdays, which may be because people make more recreational or chained trips on Friday evenings for which they seek transit information. On the weekends, there were multiple small peaks in Transit App usage distributed relatively equally during the day. The day of the week analysis revealed that overall utilization of Transit App was highest on Mondays, whereas usage of the trip planning feature peaked on Fridays, which may be because of additional non-commute trips by 
app users. On Sundays, usage (both overall and trip planning specifically) was slightly lower, which likely reflects general trends in transit ridership.

Next, the frequency with which individual Transit App users typically check the app in a single day was assessed. The results show that approximately $60 \%$ of users utilize the trip planning feature of the app exactly once per day. This group of users might be infrequent users of the transit system or travelers making irregular trips who use the trip planning feature of the Transit App only occasionally. Examining overall utilization, the percentage of users who check the Transit App two times or more in one day is almost $80 \%$, which could imply that the majority of real-time information users check this information for their commute and also for other trips, such as shopping and recreational trips. In other words, many transit riders may become accustomed to using the app and checking real-time information for most, if not all, of their transit trips.

The second part of the analysis assessed whether Transit App is used more frequently in areas with residents of certain socioeconomic characteristics based on 2010 census data. Visualizations of mean annual household income, dominant race, and dominant age per census tract showed limited, if any, relationship with the self-reported home locations of Transit App users. Transit App usage appeared to be higher in areas with a high concentration of transit service and high accessibility to businesses and recreational activities. Calculating the Pearson correlation coefficient between socioeconomic characteristics (household income, race, and age) and the number of Transit App users' self-reported home locations divided by the total population in each census tract also showed limited, if any, correlation. This finding implies that people throughout the New York City region use Transit App regardless of dominant age, race, or ethnicity in each area. Moreover, it suggests that mobile apps are becoming increasingly common and accessible to transit passengers in all areas.

In summary, this study used a new data source to assess temporal and spatial patterns of using mobile transit information use in the New York metropolitan area. Results from the temporal analysis of Transit App usage were similar to typical temporal patterns of transit system utilization, but results from the socioeconomic analysis were somewhat surprising. Limited, if any, relationship was found between Transit App usage and the socioeconomic characteristics of the population in the study region. This may be specific to New York City, which has very high transit usage in general. However, more analysis of this dataset in other regions is recommended to assess if this trend holds elsewhere.

There are numerous areas for improvement and future research that emerged from this study. One area for improvement is to compare Transit App usage with public transit ridership data. This could be done for time of day and day of week analysis, as well as the analysis of socioeconomic characteristics, in which the number of Transit App users' home locations were normalized by population per census tract since it is difficult to determine the number of transit riders per census tract. Additionally, the area of analysis could be expanded beyond the New York region to include other metropolitan areas; this is an interesting area of study because other metropolitan areas will have different socioeconomic characteristics (e.g., income and race) and varying levels of 
transit service. Future research also is suggested to verify the accuracy of locations (latitude/longitude) of users recorded in the Transit App backend server. Assessing trends in usage of the non-transit features in Transit App, such as Uber, bike sharing, and car sharing, could be examined in the future studies. Last, future research could be conducted to determine how this dataset could be integrated into the short- and longterm transit planning process.

\section{Acknowledgments}

This research was supported in part by the City University of New York (CUNY) Collaborative Incentive Research Grant (CIRG) program. We would like to acknowledge Professor Jonathan Peters at the College of Staten Island and graduate student Adam Davidson at the CUNY Graduate Center for their feedback. We also thank Transit App for providing the data used in this study, and we are particularly grateful to Jake Sion for his comments on this paper.

\section{References}

Barbeau, S., A. Borning, and K. Watkins. 2014. "OneBusAway Multi-Region-Rapidly Expanding Mobile Transit Apps to New Cities." Journal of Public Transportation, 17(4).

Brakewood, C., S. Barbeau, and K. Watkins. 2014. "An Experiment Evaluating the Impacts of Real-time Transit Information on Bus Riders in Tampa, Florida." Transportation Research Part A: Policy and Practice, 69: 409-422.

Brakewood, C., G. Macfarlane, and K. Watkins. 2015. "The Impact of Real-time Information on Bus Ridership in New York City." Transportation Research Part C: Emerging Technologies, 53: 59-75.

Brakewood, C., F. Rojas, P. C. Zegras, K. Watkins, and J. Robin. 2015. "An Analysis of Commuter Rail Real-time Information in Boston." Journal of Public Transportation, 18(1): $1-20$.

Carrel, A., A. Halvorsen, and J. L. Walker. 2013. "Passengers' Perception of and Behavioral Adaptation to Unreliability in Public Transportation." Transportation Research Record, 2351: 153-162.

Davidson, A. 2016. "Big Data Exhaust for Origin-Destination Surveys: Using Mobile TripPlanning Data for Simple Surveying." Proceedings of the 95th Annual Meeting of the Transportation Research Board.

Dziekan, K., and A. Vermeulen. 2016. "Psychological Effects of and Design Preferences for Real-time Information Displays." Journal of Public Transportation, 9(1).

Dziekan, K., and K. Kottenhoff. 2007. “Dynamic At-stop Real-time Information Displays for Public Transport: Effects on Customers." Transportation Research Part A: Policy and Practice, 41(6): 489-501. 
Feakins, J. 2013. "Planning Like It Is 2099: The Use and Distribution of Smartphone Transit Applications in Chicago, Illinois." Master's thesis, Department of Geography, Urban and Environmental Studies, Concordia University, Montreal, Quebec, Canada.

Ferris, B., K. Watkins, and A. Borning. 2010. "OneBusAway: Results from Providing RealTime Arrival Information for Public Transit." Proceedings: CHI: 1807-1816.

Gooze, A., K. E. Watkins, and A. Borning. 2013. "Benefits of Real-Time Transit Information and Impacts of Data Accuracy on Rider Experience." Transportation Research Record, 2351: 95-103.

Hickman, D., and N. Wilson. 1995. "Passenger Travel Time and Path Choice Implications of Real-time Transit Information." Transportation Research Part C: Emerging Technologies, 3(4): 211-226.

McKenzie, B. and M. Rapino. 2011. “Commuting the United States: 2009.” American Community Survey Reports, United States Census Bureau, http://www.census.gov/ prod/2011pubs/acs-15.pdf.

Schweiger, C. 2011. "TCRP Synthesis 91: Use and Deployment of Mobile Device Technology for Real-Time Transit Information." Transit Cooperative Research Program Synthesis Project SA-25, Transportation Research Board, Washington, DC.

Tang, L., and P. Thakuriah. 2012. "Ridership Effects of Real-time Bus Information System: A Case Study in the City of Chicago." Transportation Research Part C: Emerging Technologies, 22: 146-161.

Tang, L., and P. Thakuriah. 2011. “Will Psychological Effects of Real-Time Transit Information Systems Lead to Ridership Gain?" Transportation Research Record, 2216: 67-74.

Tang, L., H. Ross, and X. Han. 2012. "Substitution or Complementarity: An Examination of the Ridership Effects of Real-time Bus Information on Transit Rail in the City of Chicago." Proceedings of the 91st Annual Meeting of the Transportation Research Board.

Transit App, http://transitapp.com/. Accessed August 1, 2015.

RStudio Team. RStudio: Integrated Development for RStudio, Inc. Boston, MA, http:// www.rstudio.com/. Accessed August 1, 2015.

United States Census Bureau. New York Partnership Shapefile Batch Download. http:// www.census.gov/geo/partnerships/pvs/partnership14v3/st36_ny.html. Accessed August 1, 2015.

United States Census Bureau. American Fact Finder. http://factfinder.census.gov/faces/ nav/jsf/pages/index.xhtml. Accessed August 1, 2015.

Watkins, K., B. Ferris, A. Borning, G. S. Rutherford, and D. Layton. 2011. “Where Is My Bus? Impact of Mobile Real-time Information on the Perceived and Actual Wait Time of Transit Riders." Transportation Research Part A: Policy and Practice, 45(8): 839-848. 
Zhang, F., Q. Shen, and K. J. Clifton. 2008. "Examination of Traveler Responses to RealTime Information about Bus Arrivals Using Panel Data." Transportation Research Record, 2082: 107-115.

\section{About the Authors}

Niloofar Ghahramani (nghahra000@citymail.cuny.edu) is a doctoral student in Transportation Engineering at the City College of New York. She holds a bachelor's degree in Civil Engineering from Iran University of Science and Technology and a master's degree in Transportation Engineering from the City College of New York.

Dr. CANDace Brakewood (cbrakewood@ccny.cuny.edu) is an Assistant Professor of Civil Engineering at the City College of New York. Her research focuses on public transportation, transportation planning, and intelligent transportation systems. She has a Ph.D. in Civil Engineering from Georgia Institute of Technology, dual Master of Science degrees in Transportation and Technology Policy from Massachusetts Institute of Technology, and a Bachelor of Science in Mechanical Engineering from Johns Hopkins University. 